\title{
Recurrence Relations for the Coefficients in Chebyshev Series Solutions of Ordinary Differential Equations
}

\author{
By T. S. Horner
}

\begin{abstract}
Systematic methods are presented for obtaining recurrence relations for the coefficients in Chebyshev series solutions of linear differential equations, of first to fourth order, with polynomial coefficients. Polynomial approximations to certain rational functions are also discussed.
\end{abstract}

1. Introduction. In Morris and Horner [10], the Chebyshev series solution of a linear fourth-order homogeneous differential equation was discussed in relation to eigenvalue problems associated with simple boundary conditions. That investigation provided a systematic method for obtaining the recurrence relation for the coefficients in a Chebyshev series solution. The ideas are now applied to the solution of both homogeneous and inhomogeneous equations of orders one to four. It is also shown how the same approach can help in obtaining Chebyshev series expansions for certain rational functions.

In the literature, there are many tables of Chebyshev expansions for mathematical functions, especially the elementary functions and the special functions. Among such tables are those of Clenshaw [3], Clenshaw and Picken [4], Abramowitz and Stegun [1], Luke [7], [8], [9] which all include references to further sources. Many of the authors solve differential equations in order to find Chebyshev expansions, and the methods are fairly standard. Nevertheless, the equations are often solved on an ad hoc basis, and the aim here is to provide the data for quick and systematic construction of recurrence relations for the Chebyshev coefficients. Indeed, the data can be used to automate the solution of equations of appropriate type, but care should be taken, for example, to investigate singular points of the equation, the convergence of the solution, and the number of terms needed for a desired accuracy. In general, any automatically generated recurrence relation should also be investigated analytically.

The equations to be solved are of the form

$$
\sum_{\beta=0}^{l} p_{\beta}(x) \frac{d^{\beta} y}{d x^{\beta}}=g(x), \quad l=1,2,3,4,
$$

with suitable initial or boundary conditions.

Received June 25, 1979.

1980 Mathematics Subject Classification. Primary 65L05, 65D20. 
$l$ is the order of the differential equation,

$p_{\beta}(x)$ is a polynomial, and for most of the problems will be quadratic,

and $g(x)$ is a continuous function, assumed to be expressed in Chebyshev series form.

The Chebyshev polynomial of the first kind, $T_{n}(x)$, is used, where

$$
T_{n}(x)=\cos \left(n \cos ^{-1} x\right) \text {. }
$$

The differential equation is solved on the interval $-1 \leqslant x \leqslant 1$, to give a series as in Eq. (2.2) below. A change of variable can lead to solutions on other intervals as will be seen in later sections.

2. The Method of Solution. The method of solution is the same as that in Morris and Horner [10], and is stated briefly with regard to the solution of a secondorder equation.

Let

$$
\begin{aligned}
& p_{2}(x)=c_{1}+c_{2} x+c_{3} x^{2} \\
& p_{1}(x)=c_{4}+c_{5} x+c_{6} x^{2} \\
& p_{0}(x)=c_{7}+c_{8} x+c_{9} x^{2}
\end{aligned}
$$

Thus, Eq. (1.1) becomes

$\left(c_{1}+c_{2} x+c_{3} x^{2}\right) \frac{d^{2} y}{d x^{2}}+\left(c_{4}+c_{5} x+c_{6} x^{2}\right) \frac{d y}{d x}+\left(c_{7}+c_{8} x+c_{9} x^{2}\right) y=g(x)$, $-1 \leqslant x \leqslant 1$

Let

$$
y(x)=\sum_{k=0}^{\infty} a_{k} T_{k}(x)=\frac{1}{2} a_{0}+a_{1} T_{1}(x)+a_{2} T_{2}(x)+\cdots,
$$

and let

$$
g(x)=\sum_{k=0}^{\infty} g_{k} T_{k}(x)
$$

The derivatives $y^{(r)}(x), r=0,1,2$, are similarly given by,

$$
y^{(r)}(x)=\sum_{k=0}^{\prime} a_{k}^{(r)} T_{k}(x)
$$

to include (2.2) when $r=0$.

The following two results are used:

$$
(2 x)^{p} T_{k}(x)=\sum_{j=0}^{p}\left(\begin{array}{l}
p \\
j
\end{array}\right) T_{k-p+2 j}(x),
$$

which is a generalization of the simple recurrence relation

$$
2 x T_{k}(x)=T_{k-1}(x)+T_{k+1}(x),
$$

and

$$
a_{k-1}^{(r)}-a_{k+1}^{(r)}=2 k a_{k}^{(r-1)}
$$


which is a consequence of the fundamental theorem of calculus, and the integral

$$
\int T_{k}(x) d x=1 / 2\left\{T_{k+1}(x) /(k+1)-\left(1-\delta_{k_{1}}\right) T_{k-1}(x) /(k-1)\right\}
$$

Whenever a subscript is negative, the interpretation is

$$
a_{-k}^{(r)}=a_{k}^{(r)}
$$

The general method for solving (2.1) is then to substitute (2.3) and (2.4) to obtain, after equating coefficients of $T_{k}(x)$, followed by repeated use of (2.5) and (2.6), the recurrence relation

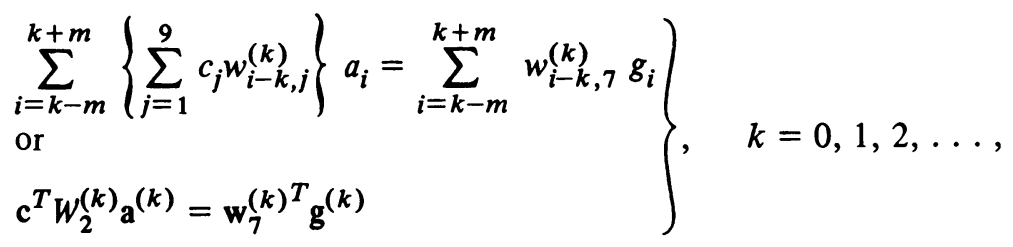

where $w_{2}^{(k)}=\left(w_{i j}^{(k)}\right)$ is the $9 \times 9$ matrix in Table 1 ,

$$
\begin{aligned}
& \mathbf{w}_{j}^{(k)} \text { is the } j \text { th column of } w_{2}^{(k)}, \\
& \mathbf{c}^{T}=\left[c_{1}, c_{2}, \ldots, c_{9}\right], \\
& \mathbf{a}^{(k)^{T}}=\left[a_{k-m}, \ldots, a_{k}, \ldots, a_{k+m}\right], \\
& \mathbf{g}^{(k)^{T}}=\left[g_{k-m}, \ldots, g_{k}, \ldots, g_{k+m}\right] .
\end{aligned}
$$

The maximum value of $m$ is 4 , but is related to the length of the vectors $\mathbf{a}^{(k)}$ and $\mathrm{g}^{(k)}$, each of which has $2 m+1$ components. Often in practice, $m$ can be taken to be less than 4 , because some of the multipliers $\left\{w_{i j}^{(k)}\right\}$ and $\left\{c_{i}\right\}$ are zero.

Once the general form of the recurrence relation is known, a suitable truncation point in the series (2.2), is chosen, (say at $a_{n}$ ), so that the series solution is sufficiently well represented by the resulting polynomial. Then the appropriate equations from (2.7), together with equations representing the initial or boundary conditions, are solved for $a_{0}, a_{1}, \ldots, a_{n}$. (See Clenshaw [2], [3], Fox and Parker [5].) This is often done by solving an explicit set of algebraic equations using standard methods such as Gaussian elimination, or iterative methods such as successive overrelaxation. A common method involves back substitution in the recurrence relation, followed by normalizing of the coefficients, using the initial or boundary conditions. The actual method for solving the algebraic equations for $\left\{a_{k}\right\}$ will not be considered further, but it is important that a stable, accurate method is selected.

Differential equations of other orders can be solved in the same manner.

Thus, let $p_{\beta}(x)$ in Eq. (1.1) be written

$$
p_{\beta}(x)=\sum_{\alpha=0}^{d_{\beta}} c_{\alpha, \beta} x^{\alpha},
$$

where $d_{\beta}$ is the degree of $p_{\beta}(x)$ and will be usually taken as 2 , but where there 


\begin{tabular}{|c|c|c|c|c|c|c|c|c|c|c|}
\hline 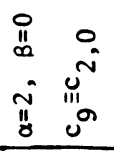 & $\overrightarrow{ \pm}$ & & S & & $\underset{\text { Tे }}{\stackrel{2}{*}}$ & & $\uparrow$ & & $\vec{i}$ & $v_{x}^{\lambda}$ \\
\hline 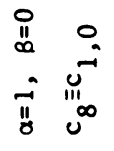 & & $\underset{\substack{I \\
\underset{N}{ \pm}}}{Z}$ & & 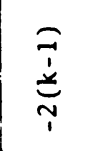 & & $\underset{\substack{I \\
\multirow{T}{*}{}}}{I}$ & & $\underset{\vec{n}}{\vec{i}}$ & & $\vec{x}$ \\
\hline $\begin{array}{ll}0 & 0 \\
0 & 0 \\
0 & 0 \\
0 & 0 \\
0 & 11 \\
0 & 0\end{array}$ & & & 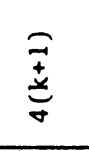 & & $\underset{0}{0}$ & & 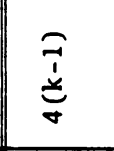 & & & $\lambda$ \\
\hline 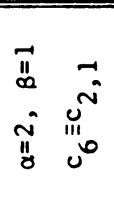 & & 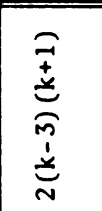 & & 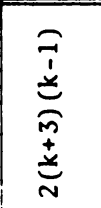 & & 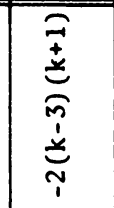 & & 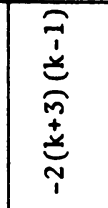 & & $\sim_{x}^{\lambda}$ \\
\hline 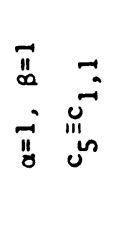 & & & $\begin{array}{l}\underset{z}{ \pm} \\
\stackrel{ \pm}{*} \\
\underset{1}{1} \\
\underset{\forall}{*}\end{array}$ & & 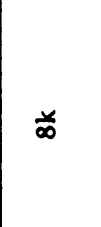 & & 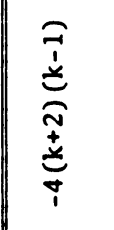 & & & $\bar{x}$ \\
\hline $\begin{array}{ll}\overrightarrow{\|} & \overrightarrow{0} \\
0 & 0 \\
0 & 0 \\
\ddot{0} & u^{\prime \prime \prime}\end{array}$ & & & & 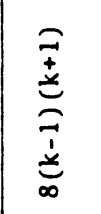 & & 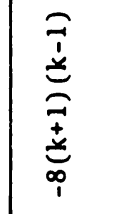 & & & & $\bar{\lambda}$ \\
\hline 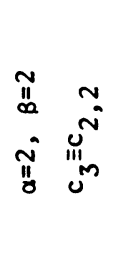 & & & 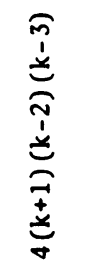 & & $\underset{\infty}{\stackrel{m}{1}}$ & & 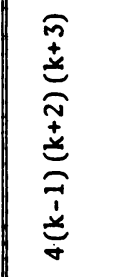 & & & $\sim_{x}^{\bar{\lambda}}$ \\
\hline 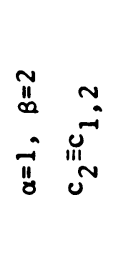 & & & & 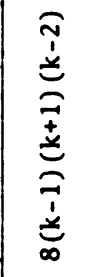 & & 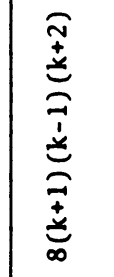 & & & & $\bar{x}$ \\
\hline $\begin{array}{cc}\pi & n \\
0 & 0 \\
0 & 0 \\
0 & 0 \\
0 & 0\end{array}$ & & & & & 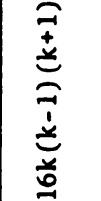 & & & & & $\bar{\lambda}$ \\
\hline & 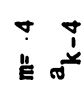 & $m \underset{m}{m}$ & 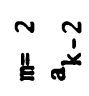 & $\vec{E}$ & 菖 & $\overrightarrow{\|}$ & 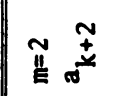 & $\underset{m}{m}$ & 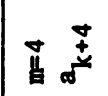 & \\
\hline
\end{tabular}


is allowance for higher degree polynomials. Now (1.1) can be written

$$
\sum_{\beta=0}^{l} \sum_{\alpha=0}^{d_{\beta}} c_{\alpha, \beta} x^{\alpha} y^{(\beta)}=g(x),
$$

where $y^{(\beta)} \equiv d^{\beta} y / d x^{\beta}$.

The recurrence relation from this equation is then found as

$$
\begin{aligned}
\sum_{i=k-m}^{k+m}\left\{\sum_{\beta=0}^{l} \sum_{\alpha=0}^{d_{\beta}} c_{\alpha, \beta} w_{i-k, \alpha, \beta}^{(k)}\right\} a_{i} \\
=\sum_{i=k-m}^{k+m} w_{i-k, 0,0}^{(k)} g_{i}, \quad k=0,1, \ldots,
\end{aligned}
$$

or

$$
\mathrm{c}^{T} W_{l}^{(k)} \mathrm{a}^{(k)}=\mathrm{w}_{0,0}^{(k)^{T}} \mathrm{~g}^{(k)} .
$$

c is the vector with elements $\left\{c_{\alpha, \beta}\right\}$, in the order of their occurrence in the differential equation (2.8).

$W_{l}^{(k)}=\left(w_{i, \alpha, \beta}^{(k)}\right)$ is a matrix of $2 m+1$ rows, and $\Sigma_{\beta=0}^{l} d_{\beta}(=\gamma$, say) columns, these columns $\left\{\mathbf{w}_{\alpha, \beta}\right\}$ being ordered to correspond to the ordering of the elements of c. The $2 m+1$ rows of $W_{l}^{(k)}$ are numbered from $-m$ to $m$, rather than from 1 to $2 m+1$, to take account of certain quasi-symmetry properties of the matrix.

The value of $m$ depends on the degrees of the polynomial coefficients $\left\{p_{\beta}(x)\right\}$, and is given by $m=\max (\alpha-\beta+l)$. When all the coefficients are quadratic, then $m=l+2$, and $\Sigma_{\beta=0}^{l} d_{\beta}=\gamma=3(l+1)$, and hence the order of $W_{l}^{(k)}$ is $(2 l+5)$ $\times(3 l+3)$.

The above notation is illustrated in Table 1 , in relation to the second-order differential equation with quadratic coefficients.

3. The Matrices $\left\{W_{l}^{(k)}\right\}$. Although it is desirable to have the matrices $\left\{W_{l}^{(k)}\right\}$ in explicit form, as in Table 1 , it is impractical to exhibit such matrices for large $l$. Thus with a modified notation for the elements of each matrix, namely writing $w_{i, \alpha, \beta}^{(k)} \equiv w(i, \alpha, \beta)$, the nonzero elements of rows 0 to $m$, are given below. If the value of any element of $W_{l}^{(k)},(l>0)$ is $f(k)$, then the element found by reflection about row 0 , is $-f(-k)$; and the entire matrix can be constructed.

The main tabulation below, corresponds to quadratic coefficients $\left\{p_{\beta}(x)\right\}$. Sometimes, further elements of $W_{l}^{(k)}$ are given at the foot of a table.

The elements are tabulated from the higher-order derivatives to the lower, but with the polynomial coefficients being written in increasing powers of $x$. Except for subsection $3.5,(l=0)$, the scaling of the elements is standardized so that there are no fractional numerical elements when all the polynomial coefficients are quadratics.

3.1. Nonzero Elements in the Lower Part of $W_{1}^{(k)} \cdot(7 \times 6)$.

$$
\begin{aligned}
& w(0,0,1)=8 k \\
& w(1,1,1)=4(k+1)
\end{aligned}
$$




$$
\begin{aligned}
& w(0,2,1)=4 k \\
& w(2,2,1)=2(k+2) \\
& w(1,0,0)=-4 \\
& w(0,1,0)=0 \\
& w(2,1,0)=-2 \\
& w(1,2,0)=-1 \\
& w(3,2,0)=-1 .
\end{aligned}
$$

The above elements correspond to quadratic coefficients $\left\{p_{1}(x), p_{0}(x)\right\}$. However, for the first-order equation all the elements of the matrix are given by simple formulas. In fact,

$$
\begin{aligned}
& w(i, \alpha, 1)=\frac{8}{2^{\alpha}}\left(\begin{array}{c}
\alpha \\
1 / 2(\alpha-i)
\end{array}\right)(k+i), \\
& w(i, \alpha, 0)=\frac{4}{2^{\alpha}}\left\{\left(\begin{array}{c}
\alpha \\
1 / 2(\alpha-i-1)
\end{array}\right)-\left(\begin{array}{c}
\alpha \\
1 / 2(\alpha-i+1)
\end{array}\right)\right\},
\end{aligned}
$$

where the combinatorial symbol $\left(\begin{array}{c}n \\ r\end{array}\right)$ is zero if $r<0, r>n$, or $r$ is noninteger.

3.2. Nonzero Elements in the Lower Part of $w_{2}^{(k)} \cdot(9 \times 9)$.

$$
\begin{aligned}
& w(0,0,2)=16 k(k-1)(k+1) \\
& w(1,1,2)=8(k-1)(k+1)(k+2) \\
& w(0,2,2)=8 k\left(k^{2}-3\right) \\
& w(2,2,2)=4(k-1)(k+2)(k+3) \\
& w(1,0,1)=-8(k-1)(k+1) \\
& w(0,1,1)=8 k \\
& w(2,1,1)=-4(k-1)(k+2) \\
& w(1,2,1)=-2(k-3)(k+1) \\
& w(3,2,1)=-2(k-1)(k+3) \\
& w(0,0,0)=-8 k \\
& w(2,0,0)=4(k-1) \\
& w(1,1,0)=-2(k+1) \\
& w(3,1,0)=2(k-1) \\
& w(0,2,0)=-2 k \\
& w(2,2,0)=-2 \\
& w(4,2,0)=k-1 \\
& w(1,3,2)=6(k+1)\left(k^{2}+k-4\right) \\
& w(3,3,2)=2(k-1)(k+3)(k+4) \\
& w(0,4,2)=6 k\left(k^{2}-5\right) \\
& w(2,4,2)=4(k+2)\left(k^{2}+2 k-5\right) \\
& w(4,4,2)=(k-1)(k+4)(k+5)
\end{aligned}
$$




$$
\begin{aligned}
& w(0,3,1)=6 k \\
& w(2,3,1)=-2(k-2)(k+2) \\
& w(4,3,1)=-(k-1)(k+4)
\end{aligned}
$$

3.3. Nonzero Elements in the Lower Part of $W_{3}^{(k)} \cdot(11 \times 12)$.

$$
\begin{aligned}
& w(0,0,3)=32 k(k-2)(k-1)(k+1)(k+2) \\
& w(1,1,3)=16(k-2)(k-1)(k+1)(k+2)(k+3) \\
& w(0,2,3)=16 k(k-2)(k+2)\left(k^{2}-7\right) \\
& w(2,2,3)=8(k-2)(k-1)(k+2)(k+3)(k+4) \\
& w(1,0,2)=-16(k-2)(k-1)(k+1)(k+2) \\
& w(0,1,2)=32 k(k-2)(k+2) \\
& w(2,1,2)=-8(k-2)(k-1)(k+2)(k+3) \\
& w(1,2,2)=-4(k-2)(k+1)\left(k^{2}-3 k-16\right) \\
& w(3,2,2)=-4(k-2)(k-1)(k+3)(k+4) \\
& w(0,0,1)=-16 k(k-2)(k+2) \\
& w(2,0,1)=8(k-2)(k-1)(k+2) \\
& w(1,1,1)=-4(k-2)(k+1)(k+5) \\
& w(3,1,1)=4(k-2)(k-1)(k+3) \\
& w(0,2,1)=-4 k\left(k^{2}-10\right) \\
& w(2,2,1)=-12(k-2)(k+2) \\
& w(4,2,1)=2(k-2)(k-1)(k+4) \\
& w(1,0,0)=12(k-2)(k+1) \\
& w(3,0,0)=-4(k-2)(k-1) \\
& w(0,1,0)=-12 k \\
& w(2,1,0)=4(k-2)(k+2) \\
& w(4,1,0)=-2(k-2)(k-1) \\
& w(1,2,0)=2(k-4)(k+1) \\
& w(3,2,0)=(k-2)(k+5) \\
& w(5,2,0)=-(k-2)(k-1) \text {. }
\end{aligned}
$$

3.4. Nonzero Elements in the Lower Part of $W_{4}^{(k)} \cdot(13 \times 15)$.

$$
\begin{aligned}
& w(0,0,4)=64 k(k-3)(k-2)(k-1)(k+1)(k+2)(k+3) \\
& w(1,1,4)=32(k-3)(k-2)(k-1)(k+1)(k+2)(k+3)(k+4) \\
& w(0,2,4)=32 k(k-3)(k-2)(k+2)(k+3)\left(k^{2}-13\right) \\
& w(2,2,4)=16(k-3)(k-2)(k-1)(k+2)(k+3)(k+4)(k+5) \\
& w(1,0,3)=-32(k-3)(k-2)(k-1)(k+1)(k+2)(k+3)
\end{aligned}
$$




$$
\begin{aligned}
& w(0,1,3)=96 k(k-3)(k-2)(k+2)(k+3) \\
& w(2,1,3)=-16(k-3)(k-2)(k-1)(k+2)(k+3)(k+4) \\
& w(1,2,3)=-8(k-3)(k-2)(k+1)(k+3)\left(k^{2}-5 k-32\right) \\
& w(3,2,3)=-8(k-3)(k-2)(k-1)(k+3)(k+4)(k+5) \\
& w(0,0,2)=-32 k(k-3)(k-2)(k+2)(k+3) \\
& w(2,0,2)=16(k-3)(k-2)(k-1)(k+2)(k+3) \\
& w(1,1,2)=-8(k-3)(k-2)(k+1)(k+3)(k+8) \\
& w(3,1,2)=8(k-3)(k-2)(k-1)(k+3)(k+4) \\
& w(0,2,2)=-8 k(k-3)(k+3)(k 2-22) \\
& w(2,2,2)=-8(k-3)(k-2)(k+2)(5 k+19) \\
& w(4,2,2)=4(k-3)(k-2)(k-1)(k+4)(k+5) \\
& w(1,0,1)=24(k-3)(k-2)(k+1)(k+3) \\
& w(3,0,1)=-8(k-3)(k-2)(k-1)(k+3) \\
& w(0,1,1)=-48 k(k-3)(k+3) \\
& w(2,1,1)=8(k-3)(k-2)(k+2)(k+5) \\
& w(4,1,1)=-4(k-3)(k-2)(k-1)(k+4) \\
& w(1,2,1)=4(k-7)(k-3)(k+1)(k+4) \\
& w(3,2,1)=2(k-3)(k-2)(k+3)(k+11) \\
& w(5,2,1)=-2(k-3)(k-2)(k-1)(k+5) \\
& w(0,0,0)=24 k(k-3)(k+3) \\
& w(2,0,0)=-16(k-3)(k-2)(k+2) \\
& w(4,0,0)=4(k-3)(k-2)(k-1) \\
& w(1,1,0)=4(k-3)(k+1)(k+8) \\
& w(3,1,0)=-6(k-3)(k-2)(k+3) \\
& w(5,1,0)=2(k-3)(k-2)(k-1) \\
& w(0,2,0)=4 k(k-19) \\
& w(2,2,0)=-(k-17)(k-3)(k+2) \\
& w(4,2,0)=-2(k-3)(k-2)(k+5) \\
& w(6,2,0)=(k-3)(k-2)(k-1) \\
& w
\end{aligned}
$$

3.5. The Matrix $w_{0}^{(k)}$. If $y$ is the rational function given by

$$
\left\{\sum_{\alpha=0}^{d_{0}} c_{\alpha, 0} x^{\alpha}\right\} y=\sum_{k=0}^{\infty} g_{k} T_{k}(x), \quad-1 \leqslant x \leqslant 1,
$$

then the Chebyshev series expansion for $y$ can be found from the recurrence relation (2.9), with the nonzero elements of $W_{0}^{(k)}$ being given by

$$
w(i, \alpha, 0)=\frac{1}{2^{\alpha}}\left(\begin{array}{c}
\alpha \\
1 / 2(\alpha-i)
\end{array}\right)
$$


4. Numerical Evaluation of $\Sigma^{\prime} a_{k}^{(r)} T_{k}(x)$. The standard evaluation for the finite sum

$$
f(x)=\sum_{k=0}^{n} a_{k} T_{k}(x)
$$

is that of Clenshaw [3].

$$
\left\{\begin{array}{l}
\text { Let } b_{n+2}=b_{n+1}=0 \\
\text { and calculate } \\
\quad b_{k}=a_{k}+2 x b_{k+1}-b_{k+2}, \quad k=n, \ldots, 1,0 . \\
\text { Then } \\
\quad f(x)=1 / 2\left(b_{0}-b_{2}\right) .
\end{array}\right.
$$

This result is easily proved using

$$
2 x T_{k}(x)=T_{k-1}(x)+T_{k+1}(x) .
$$

Clenshaw, and Smith [12] show how the numerical values of $f^{(r)}(x) / r$ ! can be evaluated using similar schemes, and Hunter [6] points out how the method is related to synthetic division for evaluating a polynomial and its derivatives.

$$
\left\{\begin{array}{l}
\text { With } b_{k+1}^{-1}=1 / 2 a_{k}, \quad k=0,1, \ldots, n, \\
\text { let } b_{n+2-r}^{r}=b_{n+1-r}^{r}=0 \\
\text { and calculate } \\
\quad b_{k}^{r}=2 b_{k+1}^{r-1}+2 x b_{k+1}^{r}-b_{k+2}^{r}, \quad k=n-r, \ldots, 1,0 . \\
\text { Then } \\
\quad \frac{f^{(r)}(x)}{r !}=1 / 2\left(b_{0}^{r}-b_{2}^{r}\right), \\
\text { for } r=0, \ldots, n .
\end{array}\right.
$$

Then (4.2) incorporates (4.1), and the values of $f$ and its derivatives can be evaluated.

At the points $x=0, \pm 1$, the particular values for $f$, and its derivatives, are

$$
\left\{\begin{array}{l}
f(0)=\sum_{i=1}^{\infty}(-1)^{i+1} a_{2 i-2} \\
f^{\prime}(0)=\sum_{i=1}^{\infty}(-1)^{i+1}(2 i-1) a_{2 i-1} \\
f^{\prime \prime}(0)=4 \sum_{i=1}^{\infty}(-1)^{i+1} i^{2} a_{2 i} \\
f^{\prime \prime \prime}(0)=4 \sum_{i=1}^{\infty}(-1)^{i+1} i(i+1)(2 i+1) a_{2 i+1}
\end{array}\right.
$$




$$
\begin{gathered}
\left\{\begin{array}{l}
f(1)=\sum_{i=0}^{\infty} a_{i}, \\
f^{\prime}(1)=\sum_{i=1}^{\infty} i^{2} a_{i}, \\
f^{\prime \prime}(1)=\frac{1}{3} \sum_{i=2}^{\infty}(i-1) i^{2}(i+1) a_{i} .
\end{array}\right. \\
\left\{\begin{array}{l}
f(-1)=\sum_{i=0}^{\infty}(-1)^{i} a_{i}, \\
f^{\prime}(-1)=\sum_{i=1}^{\infty}(-1)^{i+1} i^{2} a_{i}, \\
f^{\prime \prime}(-1)=\frac{1}{3} \sum_{i=2}^{\infty}(-1)^{i}(i-1) i^{2}(i+1) a_{i} .
\end{array}\right.
\end{gathered}
$$

For many simple applications the values of a solution and its derivatives at $x=0, \pm 1$ are sufficient for implementing initial and boundary conditions. However, the author has successfully used (4.1), (4.2) with the back-substitution method, to impose conditions at any points.

5. Examples. As foreshadowed in the Introduction, the existence of the matrices $\left\{W_{l}^{(k)}\right\}$ removes the need for a separate approach whenever a differential equation with simple polynomial coefficients is solved in Chebyshev series. As examples, expansions are obtained for $\partial J_{\nu}(x) / \partial \nu$, the derivative of the Bessel function with regard to order, and for the integral $\int_{0}^{x} J_{\nu}(t) d t$. Expansions for $J_{\nu}(x), J_{\nu}^{\prime}(x)$ are also obtained as steps in the calculations.

In order, these functions have series expansions:

$$
\begin{gathered}
J_{\nu}(x)=(1 / 2 x)^{\nu} \sum_{k=0}^{\infty}(-1)^{k}(1 / 2 x)^{2 k} /[k ! \Gamma(\nu+k+1)] \\
\frac{\partial}{\partial \nu} J_{\nu}(x)=J_{\nu}(x) \ln (1 / 2 x) \\
-(1 / 2 x)^{\nu} \sum_{k=0}^{\infty}(-1)^{k}(1 / 2 x)^{2 k} \psi(\nu+k+1) /[k ! \Gamma(\nu+k+1)] \\
\int_{0}^{x} J_{\nu}(t) d t=(1 / 2 x)^{\nu+1} 2 \sum_{k=0}^{\infty}(-1)^{k}(1 / 2 x)^{2 k} /[k !(\nu+2 k+1) \Gamma(\nu+k+1)]
\end{gathered}
$$

$\Gamma(\nu), \psi(\nu)$ are the gamma and digamma functions, respectively.

5.1. $J_{\nu}(x)$ and $\partial J_{\nu}(x) / \partial \nu$. With suitable initial conditions, $J_{\nu}(x)$ is a solution of the differential equation

$$
x^{2} y^{\prime \prime}+x y^{\prime}+\left(x^{2}-\nu^{2}\right) y=0 .
$$


Writing $J_{\nu}(x)=(1 / 2 x)^{\nu} u_{\nu}(x)$, then

$$
\frac{\partial}{\partial \nu} J_{\nu}(x)=(1 / 2 x)^{\nu}\left\{u_{\nu}(x) \ln (1 / 2 x)+\frac{\partial}{\partial \nu} u_{\nu}(x)\right\}=J_{\nu}(x) \ln (1 / 2 x)+(1 / 2 x)^{\nu} \frac{\partial}{\partial \nu} u_{\nu}(x) \text {. }
$$

Hence, on comparing this with (5.2),

$$
\frac{\partial}{\partial \nu} u_{\nu}(x)=-\sum_{k=0}^{\infty}(-1)^{k}(1 / 2 x)^{2 k} \psi(\nu+k+1) /[k ! \Gamma(\nu+k+1)] .
$$

Writing $u \equiv u_{\nu}(x), w \equiv \partial u_{\nu}(x) / \partial \nu$, then $u$ and $w$, which are even functions of $x$, satisfy the differential equations

$$
x u^{\prime \prime}+(2 v+1) u^{\prime}+x u=0,
$$

with $u(0)=1 / \Gamma(\nu+1),\left[u^{\prime}(0)=0\right]$, and

$$
x w^{\prime \prime}+(2 v+1) w^{\prime}+x w=-2 u^{\prime}
$$

with $w(0)=-\psi(\nu+1) / \Gamma(\nu+1),\left[w^{\prime}(0)=0\right]$.

An equation satisfied by the odd function $u^{\prime}(=d u / d x)=v$, is

$$
x v^{\prime \prime}+2(\nu+1) v^{\prime}+x v=-u
$$

with $[v(0)=0], v^{\prime}(0)=-1 / 2 / \Gamma(\nu+2)$.

The series

$$
\begin{gathered}
u(x)=\sum_{k=0}^{\infty}{ }^{\prime} \alpha_{k} T_{k}(x / c), \quad v(x)=\sum_{k=0}^{\infty}{ }^{\prime} \beta_{k} T_{k}(x / c), \\
w(x)=\sum_{k=0}^{\infty} \gamma_{k} T_{k}(x / c), \quad-c \leqslant x \leqslant c,
\end{gathered}
$$

are then found, after a change of variable, $x \rightarrow c x$, by solving the equations

$$
\begin{aligned}
x u^{\prime \prime}+(2 v+1) u^{\prime}+c^{2} x u & =0, & u(0) & =1 / \Gamma(\nu+1), \\
x v^{\prime \prime}+2(\nu+1) v^{\prime}+c^{2} x v & =-c u, & v^{\prime}(0) & =-1 / 2 c / \Gamma(\nu+2), \\
x w^{\prime \prime}+(2 v+1) w^{\prime}+c^{2} x w & =-2 c v, & w(0) & =-\psi(\nu+1) / \Gamma(\nu+1),
\end{aligned}
$$

where $-1 \leqslant x \leqslant 1$.

The recurrence relations for $\left\{\alpha_{k}\right\},\left\{\beta_{k}\right\},\left\{\gamma_{k}\right\}$ are

$$
\begin{aligned}
& c^{2}(k+1) \alpha_{k-3}+(k-1)\left\{4(k+1)(k+2 \nu-1)-c^{2}\right\} \alpha_{k-1} \\
& +(k+1)\left\{4(k-1)(k-2 \nu+1)-c^{2}\right\} \alpha_{k+1}+c^{2}(k-1) \alpha_{k+3}=0, \\
& c^{2}(k+1) \beta_{k-3}+(k-1)\left\{4(k+1)(k+2 v)-c^{2}\right\} \beta_{k-1} \\
& +(k+1)\left\{4(k-1)(k-2 v)-c^{2}\right\} \beta_{k+1}+c^{2}(k-1) \beta_{k+3} \\
& =-2 c(k+1) \alpha_{k-2}+4 c k \alpha_{k}-2 c(k-1) \alpha_{k-2}, \quad k=2,4, \ldots, \\
& c^{2}(k+1) \gamma_{k-3}+(k-1)\left\{4(k+1)(k+2 v-1)-c^{2}\right\} \gamma_{k-1} \\
& +(k+1)\left\{4(k-1)(k-2 v+1)-c^{2}\right\} \gamma_{k+1}+c^{2}(k-1) \gamma_{k+3} \\
& =-4 c(k+1) \beta_{k-2}+8 c k \beta_{k}-4 c(k-1) \beta_{k-2}, \quad k=3,5, \ldots,
\end{aligned}
$$

together with the appropriate initial conditions. 
In Abramowitz and Stegun [1], $\Gamma(x), \psi(x)$ are tabulated for $x=1(0.005) 2$ and $x=1(1) 101$. However, values of $\Gamma(x), \psi(x)$, for general $x$ can be found by using a Chebyshev series for $1 / \Gamma(1+x), 0 \leqslant x \leqslant 1$, given by Clenshaw [3]. Noting that

$$
\frac{d}{d x} \frac{1}{\Gamma(1+x)}=-\frac{\psi(1+x)}{\Gamma(1+x)}
$$

then Eqs. (4.1), (4.2), and the recurrence relations

$$
\Gamma(x+1)=x \Gamma(x), \quad \psi(x+1)=\psi(x)+1 / x,
$$

lead to the values of the gamma and digamma functions for any $x$ for which the functions are defined.

5.2. The Integral $\int_{0}^{x} J_{\nu}(t) d t$. Writing $\int_{0}^{x} J_{\nu}(t) d t=(1 / 2 x)^{\nu+1} z$, then it follows that the even function $z$ satisfies

$$
\begin{gathered}
x^{2} z^{\prime \prime \prime}+(3 v+4) x z^{\prime \prime}+(2 \nu+1)(\nu+2) z^{\prime}+x^{2} z^{\prime}+(\nu+1) x z=0 \\
z(0)=2 / \Gamma(\nu+2), \quad z^{\prime}(0)=0, \quad z^{\prime \prime}(0)=-1 /[2(\nu+3) \Gamma(\nu+2)]
\end{gathered}
$$

The Chebyshev expansion

$$
z(x)=\sum_{k=0}^{\infty} \alpha_{k} T_{k}(x / c)
$$

is found by letting $x \rightarrow c x$, and solving the equation

$$
\begin{gathered}
x^{2} z^{\prime \prime \prime}+(3 \nu+4) x z^{\prime \prime}+(2 \nu+1)(\nu+2) z^{\prime}+c^{2} x^{2} z^{\prime}+(\nu+1) c^{2} x z=0, \\
z(0)=2 / \Gamma(\nu+2), \quad z^{\prime}(0)=0, \quad z^{\prime \prime}(0)=-c^{2} /[(\nu+3) \Gamma(\nu+2)], \quad-1 \leqslant x \leqslant 1 .
\end{gathered}
$$

The recurrence relation is

$$
\begin{aligned}
& c^{2}(k+1)(k+2)(k+\nu-3) a_{k-4} \\
& \quad+2(k-2)(k+2)\left\{2(k+1)(k+\nu-1)(k+2 \nu-2)-c^{2}(\nu-2)\right\} a_{k-2} \\
& \quad+2 k\left\{4(k-2)(k+2)\left(k^{2}-2 \nu^{2}+\nu-1\right)-c^{2}\left(k^{2}+3 \nu-7\right)\right\} a_{k} \\
& +2(k+2)(k-2)\left\{2(k-1)(k-\nu+1)(k-2 v+2)+c^{2}(\nu-2)\right\} a_{k+2} \\
& +c^{2}(k-1)(k-2)(k-\nu+3) a_{k+4}=0, \quad k=4,6, \ldots .
\end{aligned}
$$

Conclusion. The matrices $\left\{W_{l}^{(k)}\right\}$ have been presented as a means of quickly establishing the recurrence relations for the coefficients in the Chebyshev series solutions of certain differential equations.

Second- and third-order equations were solved in the examples, and first- and fourth-order equations can be solved in a similar way. The examples were of initial value type, but boundary value conditions can be simply applied.

Linear differential equation eigenvalue problems can be formulated algebraically as in [10], but shooting methods can be used successfully for both linear and nonlinear problems. A subsequent paper will report on these problems. 
Special mention must be made of Miss Anne Cassidy who, as holder of the Austin Keane Vacation Scholarship, checked and extended the matrices $\left\{W_{l}^{(k)}\right\}$ and obtained many of the recurrence relations. Grateful thanks are also due to Miss Kerrie Griffin who did her usual excellent job in typing the manuscript. Needless to say, any errors and omissions are the fault and responsibility of the author.

Department of Mathematics

The University of Wollongong

Wollongong N.S.W. 2500, Australia

1. M. ABRAMOWITZ \& I. A. STEGUN, Handbook of Mathematical Functions, Nat. Bur. Standards, Appl. Math. Series No. 55, U. S. Government Printing Office, Washington, D. C., 1965.

2. C. W. CLENSHAW, "The numerical solution of linear differential equations in Chebyshev series," Proc. Cambridge Philos. Soc., v. 53, 1957, pp. 134-149.

3. C. W. CLENSHAW, "Chebyshev series for mathematical functions" in National Physical Laboratory Mathematical Tables, Vol. 5, Her Majesty's Stationery Office, London, 1962.

4. C. W. CLENSHAW \& SUSAN M. PICKEN, "Chebyshev series for Bessel functions of fractional order" in National Physical Laboratory Mathematical Tables, Vol. 8, Her Majesty's Stationery Office, London, 1966.

5. L. FOX \& I. B. PARKER, Chebyshev Polynomials in Numerical Analysis, Oxford Univ. Press, London and New York, 1968.

6. D. B. HUNTER, "Clenshaw's method for evaluating certain finite series," Comput. J., v. 13,1970 , pp. $378-381$.

7. Y. L. LUKE, The Special Functions and their Approximations, Vols. 1, 2, Academic Press, New York, 1969.

8. Y. L. LUKE, Mathematical Functions and their Approximations, Academic Press, New York, 1976.

9. Y. L. LUKE, Algorithms for the Computation of Mathematical Functions, Academic Press, New York, 1978.

10. A. G. MORRIS \& T. S. HORNER, "Chebyshev polynomials in the numerical solution of differential equations," Math. Comp., v. 31, 1977, pp. 881-891.

11. J. L. SCHONFELDER, "Chebyshev expansions for the error and related functions," Math. Comp., v. 32, 1978, pp. 1232-1240.

12. F. J. SMITH, "An algorithm for summing orthogonal polynomial series and their derivatives, with applications to curve-fitting and interpolation," Math. Comp., v. 19, 1965, pp. 33-36. 